\title{
Mapping non-radial pulsation using surface imaging techniques
}

\author{
S.V. Berdyugina ${ }^{1,2}$, H. Korhonen ${ }^{3}$, J.H. Telting ${ }^{4}$, C. Schrijvers ${ }^{5}$ \\ ${ }^{1}$ Institute of Astronomy, ETHZ, CH-8092 Zurich, Switzerland \\ 2 Astronomy Division, University of Oulu, P.O. Box 3000, FIN-90401 Oulu, Finland \\ 3 Astrophysikalisches Institut Potsdam, An der Sternwarte 16, D-14482 Potsdam, Germany \\ ${ }^{4}$ Nordic Optical Telescope, Apartado 474, E-38700 S/C de La Palma, Canarias, Spain \\ ${ }^{5}$ Astronomical Institute Anton Pannekoek, University of Amsterdam, Kruislaan 403, NL-1098 SJ \\ Amsterdam, Netherlands
}

\begin{abstract}
We apply stellar surface imaging techniques for studying non-radial pulsations. Stellar surface imaging is based on inversions of time series of variable spectral line profiles without making assumptions on the specific shape of the pulsations. The inversion results in an image of the stellar surface in which the sectoral and tesseral modes can in many cases be distinguished, and the pulsational degree $l$ and the azimuthal order $|m|$ can be determined.

We apply this method to simulated data for testing the method (Berdyugina et al. 2003a). The tests show that the tesseral modes with $l-|m|=2$ (or any even number) can be successfully recovered. Pulsation modes that are antisymmetric with respect to the equator, i.e. when $l-|m|=1$ (or any odd number), are difficult to recover accurately.
\end{abstract}

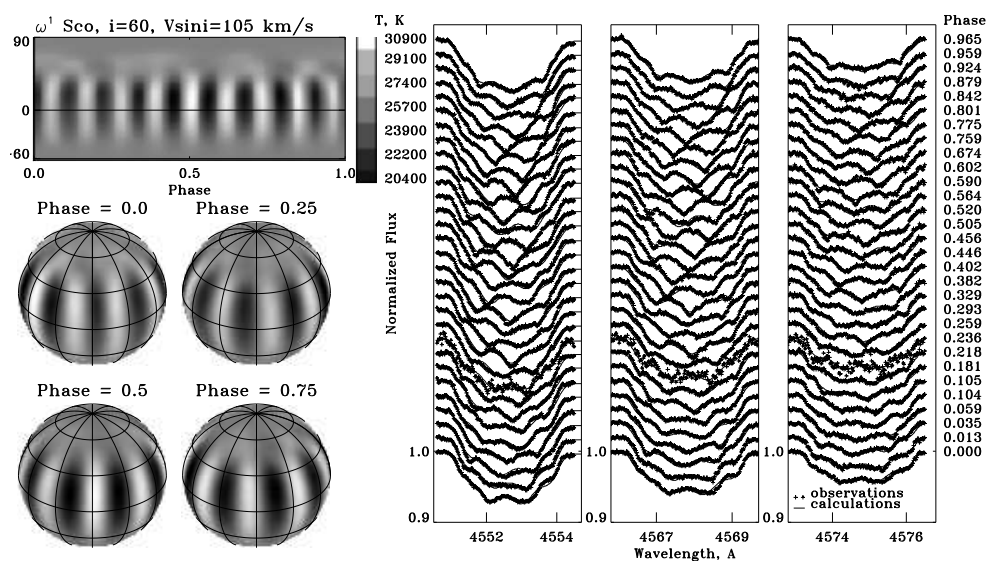

Figure 1: The image of $\omega^{1}$ Sco and fits to the observed line profiles.

The method was also applied to high-resolution spectra of the rapidly rotating Beta Ceptype star $\omega^{1}$ Sco (Berdyugina et al. 2003b). In $\omega^{1}$ Sco we find a sectoral mode of $l=|m|=9$ or $l=|m|=8$, also the tesseral mode $l=8$ with $|m|=7$ is possible. The surface map of the 
dominant pulsation mode (Fig. 1) shows amplitude modulation in the opposite hemispheres. This suggest the presence of another sectoral mode with a frequency close to that of the dominant mode.

\section{References}

Berdyugina S.V., Telting J.H., Korhonen H., 2003a, A\&A 406, 273

Berdyugina S.V., Telting J.H., Korhonen H., Schrijvers C., 2003b, A\&A 406, 281 